wurden sämtliche bestrahlten und unbestrahlten Portionen in einem regulierten Warmwasserbad 5 bis $20 \mathrm{~min}$ auf $56^{\circ} \mathrm{C}$ erwärmt, um die Trübung zu entwickeln ${ }^{3}$ ). Diese photographierten wir oder maßen sie mit dem Pulfrich-Photometer.

An den bestrahlten, alkoholfreien Portionen zeigte sich eine deutliche Trübung. 0,001 molare Alkohollösungen setzten die Trübung etwas herab, 0,01 molare und 0,1 molare verhinderten sie vollständig. Alle Alkohole wirkten bei den gleichen Konzentrationen, obwohl die narkotischen Wirkungsstärken sehr verschieden sind. Setzt man im Kaulquappenversuch die Wirkungsstärke des Äthanols $=1$, so ist sie beim n-Propanol $=4$, beim iso-Propanol $=2$, beim Butanol $=24$.

Pharmakologisches Institut der Universität, Greitswald

Eingegangen am 26. Oktober 1957

InGEBORG MEYeR

1) WEJs, P.: Pflügers Arch. 199, 226 (1923). - 2) WeLs, P., u. A. Thiele: Pflügers Arch. 209, 49 (1925). - 3) ERDMANN, K.: Naturwiss. 40, 147 (1953). - Protoplasma 45, 293 (1955). - 4) PATERSON, J., U. J. J. MATTHEWS: Nature [London] 168, 1126 (1951).5) LANGENDORFF, H., u. R. Koch: Strahlenther. 94, 411 (1954). 6) Prasliêka, M., u. J. Plesko: Českoslov. Biol. 5, 51 (1956).

\section{Schutzwirkungen einiger Alkohole an röntgenbestrahlten Eiweißlösungen}

Setzt man eine Albuminlösung einer Röntgenbestrahlung aus, so ändert sich das UV-Absorptionsspełtrum dieser Lösung im Vergleich zu einer unbestrahlten Lösung. Die Veränderung ist am einfachsten darzustellen, wenn die Spektren

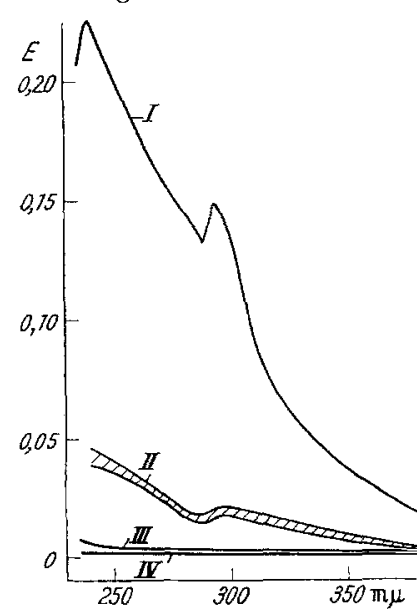
trum von bestrahltem $(80 \mathrm{~min}$ bei $1200 \mathrm{r} / \mathrm{min}$ ) gegen unbestrahltes Albumin. - II: Streubreite der Differenzspektren von bestrahltem Albumin ( $80 \mathrm{~min}$ bei $1200 \mathrm{r} / \mathrm{min}$ ) gegen unbestrahltes Albumin, beides in Gegenwart der Alkohole. III: Differenzspektrum von bestrahlten Alkoholen gegen unbestrahlte Alkohole. - IV: Differenzspektrum von Albumin unter Zusatz von Alkoholen gegen Albumin ohne Alkohole
Fig. 1. Kurve I: Differenzspekim Differenzverfahren (bestrahltes gegen unbestrahltes Eiweiß) gemessen werden. In einer früheren Arbeit wurde beschrieben, daß Trübungen, die bei Röntgenbestrahlung in Albuminlösungen entstehen, durch Zusatz verschiedener Alkohole verhindert werden können $\left.{ }^{1}\right)$. Es erhob sich die Frage, ob auch das Absorptionsspektrum diese Schutzwirkung der Alkohole wiedergibt.

Kristallines Pferdealbumin wurde $0,125 \% \mathrm{ig}$ in einer $0,8 \%$ igen $\mathrm{NaCl}-\mathrm{Lösung}$ gelöst. Vor der Bestrahlung zugesetzt wurden: Die einwertigen Alkohole Äthanol $\mathrm{n}$ - und iso-Propanol, Butanol und Pentanol; die mehrwertigen Alkohole Äthylenglykol und Glycerin. Die Konzentration dieser Alkohole war in allen Eiweißlösungen 0,01 molar. Die Strahlendosis betrug etwa. $10^{5} \mathrm{r}$ bei einer Röhrenspannung von $160 \mathrm{kV}$.

Zunächst zeigt ein Vergleich der Kurven I und II in Fig. 1 , daß die obenge nannten Alkohole, vor der Bestrahlung zugesetzt, die durch die Bestrahlung hervorgerufene Veränderung des Absorptionsspektrums vermindern. Kurve II liegt wesentlich tiefer als Kurve I. Ferner zeigt diese Kurve, daß alle angewandten Alkohole mit nur geringer Streubreite gleich stark wirken obwohl ibre narkotische Wirksamkeit weit verschieden ist. Es besteht nur der durch die Strichelung der Doppelkurve II dargestellte Unterschied. Ferner ist bemerkenswert, daß die Kurven I und II einander ähnlich sind. - Kurve IIr zeigt, daB die zugesetzten Alkohole in reiner Lösung (ohne Eiwei $B$ ) durch die Röntgenbestrahlung keine wesentliche Anderung ihres Absorptionsspektrums erfahren. Aus Kurve IV geht hervor, dab der Zusatz der Alkohole das Absorptionsspektrum der Albuminlösungen nicht merklich ändert. Die Alkohole haben in der angewandten Konzentration in dem Wellenlängengebiet von 240 bis $380 \mathrm{~m} \mu$ keine Eigenabsorption.

Pharmakologisches Institut der Universität, Greifswald INGEBORG MEYER und WILlI GRAF Eingegangen am. 26. Oktober 1957

1) Meyer, I.: Naturwiss. 44, 635 (1957).

\section{$\mathrm{CO}_{2}$-Assimilation und Einbau des Kohlenstoff} in Aminosäuren bei Pediococcus cerevisiae

Vor einiger Zeit wurde gezeigt, dab Pediococcus cerevisiae BALCKrE für den Stoffwechsel Kohlendioxyd benötigt 1). Diese Befunde konnten mittels radioaktiven $\mathrm{C}^{14} \mathrm{O}_{2}$ bestätigt und die Aufnahme von $\mathrm{CO}_{2}$ in die Körpersubstanz dieser Bakterien nachgewiesen werden ${ }^{2}$ ). Nunmehr konnte gezeigt werden, daB diese Pediokokken das C-Gerüst einiger Aminosäuren durch die Fixierung des $\mathrm{CO}$ über die Wood-WerkmanReaktion synthetisieren. Von den in Bier in Gegenwart von $\mathrm{C}^{14} \mathrm{O}_{2}$ gezüchteten Pediokokken wurden nach HCl-Hydrolyse 15 Aminosäuren mit der chromatographischen Verteilungsanalyse identifiziert. Von diesen hatten funf den markierten Kohlenstoff eingebaut. Stark radioaktiv zeigten sich nach dem Autoradiogramm: Asparaginsäure und Glutaminsäure, außerdem Lysin. Wesentich schwächer radioaktiv waren Serin und Arginin.

Institut für Gürungsphysiologie und. Technische Mikrobiologie der Technischen Hochschule, Mïnchen-Weihenstephan

Eingegangen am 30. Oktober 1957

F. WEINFURTNER

1) Weinfurtiner, F., A. Uhl u. R. Pöhlmann: Brauwiss. 8, 166, 192 (1955). - $\left.{ }^{2}\right)$ Weinfurtner, F.: Brauwiss, 10, 127 (1957).

\section{Zur Frage des endogenen Substrats} und der Glukose-Hemmung der Atmung

Der endogene Zellstoffwechsel bietet eine Reihe von ungelösten Problemen; insbesondere ist die chemische Natur des veratmeten Substrats und die Beziehung $z$ wischen den endogenen und den exogenen Substraten ungeklärt. Schon WAR$\mathrm{BURG}^{1}$ ) bemerkte, daß sich der Retikulozyt durch eine lang-

Tabelle 1. $O_{2}$-Verbrauch und $N H_{3}$-Bildang in Retikulosyten und in Aszites-Tumorzellen unter endogenen Bedingungen (0,9\% $\mathrm{NaCl}$ ) und in Gegenwart von Glukose $(200 \mathrm{mg} / 100 \mathrm{ml})$

\begin{tabular}{|c|c|c|c|c|c|c|}
\hline & \multicolumn{3}{|c|}{ Retikulozyten } & \multirow{2}{*}{\multicolumn{3}{|c|}{$\frac{\text { Aszites-Tumorzellen }}{\mu \mathrm{Mol} / \mathrm{ml} \text { Zellen } / 2 \text { Std }}$}} \\
\hline & \multicolumn{3}{|c|}{$\mu \mathrm{Mol} / \mathrm{ml}$ Zellen/4 Std } & & & \\
\hline & $\triangle \mathrm{O}_{2}$ & $\Delta \mathrm{NH}_{3}$ & $\left.v^{*}\right)$ & $\Delta \mathrm{O}_{2}$ & $\triangle \mathrm{NH}_{3}$ & $\left.v^{*}\right)$ \\
\hline$\underset{\mathrm{NaCl}}{\mathrm{NaCl}}+\dot{\text { Gilukose }}$ & $\begin{array}{l}-25,2 \\
-21,6\end{array}$ & $\begin{array}{l}+3,57 \\
+2,32\end{array}$ & $\begin{array}{l}7,1 \\
9,3\end{array}$ & $\begin{array}{l}-107 \\
-48,8\end{array}$ & $\begin{array}{l}+15,2 \\
+\quad 5,2\end{array}$ & $\begin{array}{l}7,0 \\
9,4\end{array}$ \\
\hline
\end{tabular}

anhaltende endogene Atmung auszeichnet. Die Frage nach dem veratmeten Substrat blieb offen. Aus unseren Beobachtungen geht hervor, daß die endogene Atmung zumindest teilweise auf die Verbrennung von Aminosäuren zurückgeführt werden mnß. Dafür spricht 1. eine proportionale Beziehung zwischen Ammoniakbildung und Sauerstoffverbrauch, wobei die Nukleotide keine wesentliche Desaminierung erfahren; 2. ein analoges Verhalten von Sauerstoffver brauch und Ammoniakbildung bei verschiedenen $\mathrm{Di}$ nitrophenolkonzentrationen und 3. als direkter Beweis die Tatsache, daß bei Zusatz von Indikatormengen von Glyzin-1-14 C Kohlendioxyd ${ }^{14} \mathrm{CO}_{2}$ gebildet wird.

Auch Asciteszellen haben

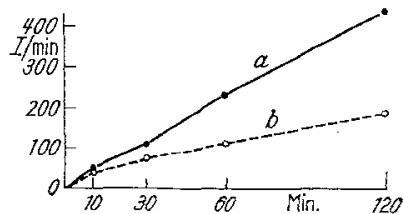

Fig. 1. Spezifische Aktivität des Atmungs-CO von Ascitestumorzellen in Gegenwart von Glyzin-

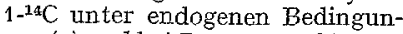
gen $(a)$ und bei $Z$ usat $z$ von Glukose (b), gemessen als $\mathrm{BaCO}_{3}$ bei unendlicher Schichtdicke. a Ohne, $b$ mit Glukose eine starke endogene At-

mung. Auf Grund analoger Untersuchungen kommen wir zu dem Schluß, daß unter endogenen Bedingungen auch Asciteszellen zumindest teilweise Aminosäuren veratmen.

Zusatz von Glukose hemmt sowohl in Retikulozyten als auch in Tumorzellen (Tabelle 1) in annähernd gleicher Weise Sauerstoffverbrauch und Ammoniakbildung; dies geht aus dem $\mathrm{O}_{2} / \mathrm{NH}_{3}$-Quotienten hervor. Bei $\mathrm{Zusatz}$ von Glukose wird die spezifische Aktivität des gebildeten $\mathrm{CO}_{2}$ herabgesetzt (Fig. 1). Bei Tumorzellen sind die Glukose-Effekte größer. Eine Hemmung der Ammoniakbildung bei Asciteszellen wurde schon von WARBURG ${ }^{2}$ ), die der Sauerstoffaufnabme von CRABTreE ${ }^{3}$ ) beobachtet. Die Wirkung auf die Sauerstoffaufnahme wurde auch an anderen Zellen beobachtet ${ }^{4}$. 